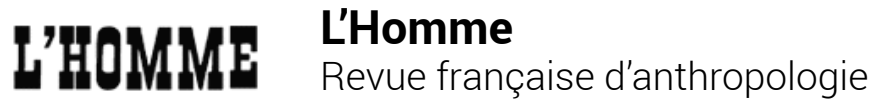

175-176 | juillet-septembre 2005

Vérités de la fiction

\section{Jan Petrus Benjamin De Josselin de Jong, Les Danses des Piegan : danses indiennes d'Amérique du Nord}

Strasbourg, Impasses de l'Encre, 2004, 60 pages

\section{Emmanuel Désveaux}

\section{OpenEdition}

\section{Journals}

Édition électronique

URL : http://journals.openedition.org//homme/2062

DOI : 10.4000//homme.2062

ISSN : 1953-8103

Éditeur

Éditions de l'EHESS

Édition imprimée

Date de publication : 15 octobre 2005

Pagination : 522-524

ISBN : 2-7132-2035-1

ISSN : 0439-4216

\section{Référence électronique}

Emmanuel Désveaux, « Jan Petrus Benjamin De Josselin de Jong, Les Danses des Piegan : danses

indiennes d'Amérique du Nord », L'Homme [En ligne], 175-176 | juillet-septembre 2005, mis en ligne le 30 novembre 2006, consulté le 23 septembre 2020. URL : http://journals.openedition.org//homme/2062 ; DOI : https://doi.org/10.4000//homme.2062

Ce document a été généré automatiquement le 23 septembre 2020

(C) École des hautes études en sciences sociales 


\title{
Jan Petrus Benjamin De Josselin de Jong, Les Danses des Piegan : danses indiennes d'Amérique du Nord
}

\author{
Strasbourg, Impasses de l'Encre, 2004, 60 pages
}

\section{Emmanuel Désveaux}

\section{RÉFÉRENCE}

Jan Petrus Benjamin De Josselin de Jong, Les Danses des Piegan : danses indiennes d'Amérique du Nord. Trad. du néerlandais sous la dir. de Thomas Beaufils. Strasbourg, Impasses de l'Encre, 2004, $60 \mathrm{p}$.

TOUT LE MONDE connaît le nom de Jan Petrus Benjamin De Josselin de Jong, grande figure de l'ethnologie indonésienne et à qui la tradition anthropologique attribue le titre envié de précurseur du structuralisme. On a tendance à oublier qu'avant de faire du terrain au cœur de l'empire colonial de son pays, l'éminent professeur de l'université de Leyde a commencé sa carrière comme américaniste. D'aucuns se souviennent vaguement de sa - brillante - intervention dans la discussion du problème natchez (mais qui s'intéresse encore au problème natchez aujourd'hui?), tandis que les spécialistes du champ ont eu l'occasion de consulter ses recueils en langue originale de textes ojibwa. En revanche, nous devons avouer que sa contribution à l'ethnographie des Blackfoot nous avait échappé. Ce qui se comprend puisqu'elle se limite à la publication, en 1912 et en néerlandais, d'un bref article d'une quarantaine de pages qui n'avait jamais été traduit ni en français, ni même, semble-t-il, en anglais ${ }^{1}$. Saluons donc l'initiative de Thomas Beaufils qui nous en propose une élégante traduction française. L'entreprise est d'autant plus digne d'attention que cette production a été l'occasion d'une action pédagogique : ce travail de traduction a été mené collectivement sous sa direction par un groupe d'étudiants du département d'études néerlandaises de l'université Marc-Bloch à Strasbourg. 
2 Ainsi que l'explique Thomas Beaufils dans sa brève introduction, De Josselin de Jong se rend à vingt-cinq ans dans le Montana chez les Piegan, sous-groupe des Indiens Blackfoot. Nous ignorons s'il s'y rend en compagnie de son professeur, C. C. Uhlenbeck, ou si c'est seulement à l'initiative de celui-ci. Il est certain en revanche que ce déplacement jusqu'aux confins des Rocheuses est lié au travail de terrain déjà entrepris par ce dernier. D'ailleurs, c'est Uhlenbeck qui deviendra le grand spécialiste des Blackfoot et de leur langue, puisqu'il publiera dans les années 1920 et 1930 tour à tour un dictionnaire, une grammaire et un recueil de mythes les concernant. Qualifions donc la présence du jeune De Josselin chez les Blackfoot de ce qu'on appellerait aujourd'hui un terrain didactique et apprécions pleinement ce qu'il en ressort.

3 L'article, destiné à l'origine au public assez large de la revue Onze eeuw (« Notre siècle ») qui le publia, est consacré aux principales danses ayant cours chez les Piegan au moment de l'enquête, à savoir la danse de l'herbe (grass dance) adopté des Sioux, la danse du calumet ou de la pipe sacrée, la danse du castor, mais dont finalement il ne soufflera pas un mot, et la danse du soleil. Après avoir évoqué les contours de la famille linguistique algonquine à laquelle appartiennent les Blackfoot et avoir souligné combien il serait vain d'en reconstituer la généalogie faute de connaissances historiques suffisantes, De Josselin commence par traiter de la danse de l'herbe. Il la décrit - reconnaissons qu'il cède ici au goût du pittoresque, en particulier lorsqu'il parle des danseurs et de leur accoutrement - et en rapporte le mythe d'origine. Le fait que cette danse ne soit pas liée au cycle calendaire, ni ne réponde à un impératif rituel clairement identifié le conduit à penser qu'elle possède un caractère profane et non sacré, comme le seraient inévitablement les trois autres. On perçoit l'influence de Durkheim, pas nécessairement bienvenue. Mais il s'agit pour lui d'affirmer qu'en dépit des apparences, les Indiens savent aussi uniquement se distraire.

4 La section sur la danse du calumet en relate le mythe d'origine. Puis, plus que la danse elle-même, l'auteur se concentre sur l'ethnographie de la pipe. Il en donne une description très précise, ainsi que celle de son fourreau, se déployant sur une page entière et portant sur la taille des divers éléments, les matériaux, les accessoires ; ainsi que, nous renseigne-t-il, sur le nombre de perles accrochées, le nombre de lanières utilisées, etc. Dans la mesure où De Josselin reconnaît candidement n'avoir jamais vu l'objet en question, cela représente un véritable tour de force... de son ou ses informateurs. La pipe appartient à toute la tribu. Elle a vocation à circuler entre de nombreuses mains et son culte tient à un double cycle, l'un régulier, saisonnier, l'autre aléatoire, lié à la maladie. Si quelqu'un tombe malade, il prête serment d'organiser, en cas de guérison, une fête en l'honneur de la pipe. Si cette guérison intervient, une fête est donnée, la danse est alors menée par la personne guérie qui, du même coup, entre en possession de la pipe. Selon maintenant une autre temporalité, lors d'une cérémonie à caractère cyclique qui se tient à l'orée de l'hiver, la pipe est également exhibée. Tous les anciens détenteurs de la pipe y sont invités, les quatre plus récents devant battre le tambour. Ensuite, la pipe ne quitte plus son fourreau de tout l'hiver, sauf, comme nous l'avons vu, circonstances particulières dues à l'irruption d'une maladie chez un tel ou un tel. Enfin, au premier coup de tonnerre printanier, une cérémonie fait écho à celle de l'automne qui en reprend le déroulement. Pour notre part, dans le prolongement de notre théorie des socièmes élémentaires ${ }^{2}$, nous retiendrons le caractère confrérique de ce double dispositif cérémoniel dont fait l'objet la pipe sacrée. Nous avons bien affaire à un groupe d'individus élus, en l'occurrence élus par la maladie, ou plus exactement par 
la survie à celle-ci, qui se dissout et se recompose en fonction de l'alternance saisonnière.

5 La dernière partie du texte est consacrée à la danse du soleil. C'est la plus complexe et la plus argumentative. S'inspirant dans un premier temps de Clark Wissler et de sa vaste enquête comparative sur les danses du soleil, De Josselin tente d'abord de définir un prototype rituel commun à toutes les tributs algonquines des Plaines pour annoncer dans un second temps que la forme blackfoot apparait d'emblée comme trop divergente pour que le schéma global soit convainquant. Si le rituel, appelé Medecine-Lodge chez les Blackfoot, comporte effectivement l'érection rituelle d'un mât et la construction autour de lui d'une tonnelle, ainsi que diverses offrandes à l'astre du jour, il ne repose pas sur des séances d'autotorture masculine comme chez les Sioux, ou chez les Cheyennes, synonyme d'automutilation, mais sur la non-sexualité supposée d'une femme. En effet, l'ensemble de la cérémonie s'orchestre autour d'une femme qui a formulé un vœu en faveur de la tenue du rituel, et qui, ensuite, y tient le rôle central, éventuellement en compagnie de son mari. Or, la valeur de ce vœu est solidaire de la chasteté de son énonciatrice. Une fois encore, nous voyons se dessiner ici les linéaments d'un vaste système transformationnel dont nous avons développé l'analyse ailleurs ${ }^{3}$. Sans prétendre que De Josselin anticipe ces résultats, nous avons le plaisir de le lire lorsque, à peine frais émoulu de l'université, il attaque fortement la méthode du diffusionnisme historique que défendait alors sur les mêmes thèmes Wissler, le monstre sacré de l'époque. Que ce soit à propos de la danse de la pipe ou celles dites du soleil, il est impossible aux yeux du jeune Hollandais de reconstituer leur généalogie et de déterminer une forme qui précéderait une autre, a fortiori avec l'espoir de remonter jusqu'à celle à l'origine de toutes les autres. Il est tout aussi impossible d'assigner une forme rituelle à une langue, en l'occurrence l'algonquin et le sioux, puisque, précisément dans les Plaines, se rencontrent des formes rituelles ressemblantes, mais jamais similaires, de façon indifférente chez les locuteurs de l'une et chez ceux de l'autre. Une "culture " ne peut se définir en conséquence en vertu d'une étroite homogénéité entre une langue et des traits comportementaux et de leur évolution conjuguée au cours de l'histoire.

6 Il est toujours tentant de déceler a posteriori les qualités intrinsèques d'une œuvre dans ses toutes premières manifestations. Nous nous en garderons donc. Notons toutefois que De Josselin de Jong avait été formé à la méthode génétique, avec pour arrière-fond les études indo-européennes. Le contact avec les données américaines, foncièrement rétives à cette méthode, l'a convaincu très tôt et de façon plus ou moins inconsciente de l'existence d'autres modalités d'explication d'une réalité empirique donnée, la relation contrastive entre deux termes étant, nous le savons depuis Jakobson, la principale d'entre elles. En bref, émettons l'hypothèse que le protostructuralisme du grand maître de Leyde tient autant à son expérience américaine initiale qu'à ses terrains indonésiens postérieurs à propos duquel il trouvera pourtant à s'exprimer explicitement.

7 Une ultime remarque sur l'aspect précurseur de ce texte. On prête à Malinowski l'invention théorique de l'observation participante dans les années 1920. Lisons simplement cette phrase, rédigée par De Josselin en 1912: «Il m'est impossible de rendre compte de l'entière signification religieuse de cette danse, d'esquisser sa relation vis-à-vis de l'ancien rituel et, ce faisant, de donner une image vivante de la vie religieuse née de cette influence réciproque. Pour y parvenir, une expérience 
personnelle ainsi qu'un profond sentiment d'appartenance sont indispensables - et ces conditions me font défaut... » (p. 31).

\section{NOTES}

1. . Si l'on en juge d'après l'impressionnante bibliographie du vol. 13 du Handbook of North American Indians, consacré aux Plaines sous la responsabilité de Raymond J. DeMallie (William C. Sturtevant, ed., Washington, Smithsonian Institution, 2001).

2. .Emmanuel Désveaux, Quadratura americana, essai d'anthropologie lévi-straussienne, Genève, Georg, 2001.

3. .Cf. Emmanuel Désveaux, "Guerre et mariage, confréries et clans : aperçu sociologique et rituel », in Michel Le Bris, ed., Indiens des Plaines: les peuples du bison, Paris, Hoëbeke, 2000 : 111-127. 